\title{
A teoria do U invertido: um teste da hipótese de Kuznets para a relação entre crescimento econômico e desigualdade de renda no Brasil (1976-2007)
}

\author{
Wesley Oliveira Santos ${ }^{1}$ \\ Fábio Rodrigues de Moura ${ }^{2}$ \\ Anderson Renê Santos Silva ${ }^{3}$ \\ Danilo Lima Matos ${ }^{4}$ \\ Tácito Augusto Farias ${ }^{5}$
}

Resumo: O presente artigo examina a relação entre crescimento econômico e desigualdade na distribuição de renda no Brasil, com o intuito de verificar, para o país, a aplicabilidade da hipótese do "U-invertido" formulada por Simon Kuznets (1955), a qual estabelece que a referida relação segue um padrão onde a desigualdade é crescente nos primeiros estágios de desenvolvimento até um ponto de inflexão, a partir do qual a mesma passa a cair ao longo da ocorrência do crescimento econômico. Mediante aplicação de um modelo econométrico auto regressivo de primeira ordem (AR1) a séries de tempo, foi possível comprovar empiricamente a teoria proposta por Kuznets, ou seja, que a relação entre desigualdade de renda e crescimento econômico para o Brasil no período 1976-2007 segue o formato de "U invertido".

Palavras-chave: desigualdade de renda; distribuição de renda; crescimento econômico; curva de Kuznets.

\footnotetext{
1 Graduado em Ciências Econômicas pela Universidade Federal de Sergipe (UFS).

2 Mestrando em Economia pela Universidade Federal do Pernanbuco (UFPE).

3 Graduando em Ciências Econômicas pela Universidade Federal de Sergipe (UFS).

4 Graduado em Ciências Econômicas pela Universidade Federal de Sergipe (UFS).

5 Doutor em Economia Aplicada pela Universidade de São Paulo (USP).
} 


\title{
“Inverted U", theory: Kuznet's hypothesis test for the relationship between economic growth and income inequality in Brazil \\ (1976-2007)
}

\begin{abstract}
This article examines the relationship between economic growth and income inequality in Brazil, in order to verify the applicability of Simon Kuznets' (1955) "inverted-U" hypothesis, which establishes that this relationship follows a pattern where inequality grows in the early stages of development to an inflection point, after which it will fall over the occurrence of economic growth.By applying a first order auto-regressive (AR1) econometric model in time-series, it was possible to empirically prove the theory proposed by Kuznets, i.e. the relationship between income inequality and economic growth follows the format of "inverted U" to Brazil in the period 1976-2007.
\end{abstract}

Keywords: income inequality; distribution; economic growth; kuznets curve.

JEL: O15; C32.

Introdução

A desigualdade na distribuição de renda nos países latino-americanos, particularmente no Brasil, é um problema historicamente não resolvido, que vem ganhando importância no campo teórico, no debate social e na pauta das principais políticas públicas em vigor no país. Segundo o Relatório de Desenvolvimento Humano 2007/2008 (publicado pelo Programa das Nações Unidas para o Desenvolvimento) o desempenho do Brasil, de acordo com o Índice de Gini de desigualdade de Renda, o situa entre os 12 países mais desiguais do mundo.

No Brasil, ao menos desde a década de 70, quando as políticas macroeconômicas passam a ter um caráter mais elaborado, os formuladores de políticas parecem pôr como prioridade máxima ou a busca de estabilidade de preços, ou o crescimento econômico, ou ainda a combinação dos dois. O objetivo distributivo na maioria das vezes (senão todas) foi deixado de lado, tomando em diversas ocasiões a forma de promessas vagas, que ainda assim expressam o caráter subsidiário do mesmo, a exemplo do jargão "primeiro deixar o bolo crescer para depois distribuir".

Se tratando de variáveis econômicas, para uma aplicação mais direcionada e eficaz de políticas públicas, é imprescindível a observação do comportamento padrão de cada uma e o exame de suas interações. Neste sentido, algumas 
perguntas são pertinentes: Existe alguma relação sistemática entre crescimento econômico e distribuição de renda? Caso exista, qual o padrão dessa relação?

$\mathrm{Na}$ investigação desta temática, não raros são os casos de resultados controversos dentro de uma mesma categoria ou em uma única subcategoria de análise. Um caso particular, que será objeto de investigação deste trabalho, é o estudo da hipótese formulada por Simon Kuznets (1955), conhecida na literatura como "Hipótese do U-invertido" ou "Curva de Kuznets", a qual estabelece uma relação em forma de U-invertido entre desigualdade na distribuição de renda e crescimento econômico.

Como será exposta ao longo deste trabalho, tanto a investigação teórica como a verificação empírica da curva de Kuznets são compostas de estudos com resultados significativamente divergentes no que toca à aceitação ou não da referida hipótese. Especificamente quanto à hipótese de Kuznets, há ainda interpretações divergentes sobre o aumento da desigualdade nos períodos iniciais do crescimento, em que uns afirmam que tal fato decorre da redução absoluta da renda média da população mais pobre enquanto outros defendem que o referido aumento é fruto apenas da menor taxa de crescimento da renda média dos mais pobres em relação à dos mais ricos, nos primeiros estágios do crescimento.

No geral, os trabalhos mais dignos de nota giram em torno de análises com dados transnacionais. Tais análises, no entanto, podem produzir resultados que retiram o caráter dinâmico da relação crescimento-desigualdade pela ausência de uniformidade de elementos estruturais e históricos, cujas interações deixam de ser captadas quando a investigação não é feita com dados de um único país e sim de um conjunto desses, onde cada um apresenta características históricas e estruturais individuais e essencialmente heterogêneas. A fim de eliminar essas limitações, neste trabalho, o poder de explicação da hipótese de Kuznets será testado mediante utilização de dados nacionais, representativos de crescimento e distribuição de renda no Brasil.

\section{Referencial teórico}

O trabalho que constitui a maior referência teórica para o presente estudo foi feito por Simon Kuznets (1955), cujo postulado ficou conhecido na literatura como "Hipótese do U-Invertido" ou "Curva de Kuznets", termos que expressam a forma geométrica da relação entre crescimento e desigualdade. Através de um estudo empírico da relação em três países (Inglaterra, EUA e Alemanha) Kuznets postulou que a desigualdade de renda aumenta nos estágios iniciais de crescimento, atingindo um ponto máximo, representado por um determinado nível de renda per capita, a partir do qual decresce à medida que a economia se desenvolve. Segundo Kuznets, o processo ocorreria por meio da migração da população de um setor primário, mais atrasado, para o 
setor urbano, mais moderno, rico e desigual que o tradicional. As mudanças na distribuição decorrentes de tal migração, característica intrínseca do crescimento econômico moderno, promoveriam um aumento inicial da desigualdade até que o produto per capita atingisse certo patamar, a partir do qual a desigualdade se estabilizaria, adquirindo, posteriormente, uma tendência de redução contínua à medida que a renda per capita permanecesse evoluiindo (Kuznets, 1982). Essa hipótese tem sido objeto de investigação teórica e empírica de diversos estudos, com interpretações divergentes e resultados controversos.

Em algumas interpretações mais pessimistas, o desenvolvimento dos países, além de apresentar incrementos na desigualdade relativa, vem acompanhado de deteriorações da renda absoluta para as classes mais pobres da população (Ahluwalia, 1976; p. 128).

A seguir estão expostas algumas abordagens relevantes acerca da literatura teórica e empírica deste postulado.

\subsection{Literatura Teórica sobre a Hipótese de Kuznets}

Acerca da investigação teórica da curva de Kuznets, a literatura pode ser classificada pela associação da referida hipótese a três grupos de modelos, os quais não necessariamente assumem a linha de investigação que estabelece que a relação ocorra na direção do crescimento influenciando o nível de desigualdade, conforme exposto no postulado original. Dessa forma, esses modelos podem estabelecer que a relação se dê na direção oposta, ou seja, com a desigualdade influenciando o crescimento econômico. Em conformidade com a divisão exposta em Jacinto e Tejada (2004), as três linhas de investigação são: i) modelos dualistas; ii) modelos de crescimento e iii) modelos de escolha social.

O primeiro grupo, de modelos dualistas, é caracterizado pela investigação da relação entre desigualdade e crescimento numa economia com dois setores, um agrícola tradicional e um setor dinâmico industrial. Neste, além do próprio trabalho de Kuznets (1955), destacam-se a formulação mais rigorosa de Robinson (1976 apud Jacinto e Tejada, 2004), que, sem supor a existência de desigualdade intra setorial no setor em crescimento, demonstrou o padrão de U invertido partindo apenas da hipótese de disparidades na renda média entre os dois setores.

O segundo grupo de tratamento teórico da hipótese de Kuznets é particularizado por estudos que partem das implicações distributivas das modernas teorias de crescimento, em particular, do modelo de crescimento econômico de Solow. Esse modelo, que supõe agentes homogêneos, é composto por uma função de produção neoclássica e por uma equação de acumulação de capital. A função de produção neoclássica, por hipótese, possui retornos constantes 
de escala e produtos marginais por insumo positivos e decrescentes. Por sua vez, a equação de acumulação de capital é obtida através da diminuição da depreciação (suposta constante) do acréscimo de capital num determinado período de tempo. No modelo básico, com agentes idênticos e supondo o crescimento populacional constante, Solow chega à conclusão da existência, no longo prazo, de uma convergência das economias dos diversos países rumo a um equilíbrio estacionário pré-determinado, com incrementos constantes do rendimento per capita, cujo nível dependeria do grau de tecnologia e das preferências. Se a renda per capita de uma determinada economia encontra-se abaixo desse nível, pode ser demonstrado que o rendimento per capita sofrerá aumentos monotônicos. O modelo de Solow, ao supor a homogeneidade dos agentes, exclui a possibilidade de influência da desigualdade no crescimento econômico (Zilberman, 2004). Se, por outro lado, a suposição de agentes idênticos for substituída pela hipótese de heterogeneidade dos mesmos, o modelo se torna capaz de demonstrar padrões de desigualdade de crescimento do rendimento per capita. Os trabalhos que constituem esforços nessa direção vêm sendo modificados nas últimas décadas, de modo a incorporar os novos avanços da literatura, como o crescimento endógeno ou as análises de equilíbrio envolvendo elementos políticos de escolha social, que constitui o terceiro grupo de investigação teórica da hipótese do U invertido.

Os modelos de escolha social admitem a existência de uma relação causal entre as decisões políticas dos eleitores - relativas a programas que proporcionem o atendimento imediato de suas necessidades - e ao vínculo entre desigualdade inicial e distribuição de renda. Esses modelos partem da suposição de um contexto de disparidade da distribuição de renda e riqueza e consideram que as decisões do governo são tomadas em função das interações políticas, fruto das aspirações da população com respeito a temas de seu interesse (como nível de impostos, por exemplo). Essas interações de interesses e as consequentes decisões governamentais são representadas através do "teorema do eleitor mediano", que funciona da seguinte forma: caso o contexto seja o de uma sociedade com níveis acentuadamente desiguais da distribuição de renda e riqueza, o eleitor mediano (desprovido de recursos) desejará obter ganhos no curto prazo, no sentido de ansiar uma distribuição de renda do capital para o trabalho. Desse modo, os governos que tendem a ser eleitos são aqueles cujos programas abordem com maior ênfase a característica distributiva. Tal repartição prejudicaria a acumulação de capital, reduzindo os níveis de investimento e prejudicandò o processo de crescimento econômico. Contudo, caso o contexto seja o de uma sociedade com uma distribuição mais igualitária de renda e riqueza, a população tenderia a eleger governos que tratassem o problema distributivo de forma secundária. Assim, a acumulação de capital e os investimentos evoluiriam, favorecendo o processo de crescimento. 


\subsection{Literatura Empírica sobre a Hipótese de Kuznets}

A literatura que tem por objeto a verificação empírica da curva de Kuznets é bastante ampla e metodologicamente diversificada. As conclusões apresentadas nos diversos trabalhos estão longe de estabelecer um consenso entre a existência ou não de evidências desta relação. Para executar essas investigações, costuma-se fazer uso de diversos índices de concentração e de desigualdade aliados à utilização de uma grande variedade de formas funcionais específicas e de métodos de estimação. Assim, na presente literatura encontram-se trabalhos que confirmam e outros que refutam a referida hipótese. De uma forma geral, até o momento, as principais contribuições da referida literatura se dão em torno das discussões relacionadas à especificação de modelos, utilização de base de dados e métodos empregados para estimar a curva de Kuznets.

Num trabalho de grande relevância, realizado por Ahluwalia (1976), utilizou-se uma amostra de 60 países (40 subdesenvolvidos, 14 desenvolvidos e 6 socialistas da Europa oriental), aplicadas a diferentes modelos. Um modelo econométrico mais geral foi especificado com o intuito de captar mudanças estruturais, presentes nos modelos dualistas. Na aplicação do modelo, 0 autor dividiu a população por faixas de renda, buscando identificar a relação entre as diversas variáveis citadas abaixo e as participações na renda total dos $40 \%$ mais pobres, dos $60 \%$ mais pobres, dos $40 \%$ médios e dos $20 \%$ mais ricos. Este modelo mais amplo possuía a seguinte forma:

$L i=\alpha+\beta_{1} \log \mathrm{Y}+\beta_{2} \log \mathrm{Y}_{2}+\beta_{3} y+\beta_{4} E+\beta_{5} M+\beta_{6} P+\beta_{7} A+\beta_{8} U+\beta_{9} S+f(1 \mathrm{a})$

Onde: $L_{i}=$ Participação na renda total. Sendo estas representadas por $i=1$, $2,3,4$, cujos índices representam, respectivamente, a participação na renda total dos $40 \%$ mais pobres, dos $60 \%$ mais pobres, dos $40 \%$ médios e dos $20 \%$ mais ricos; $\alpha=$ Intercepto; $Y=$ PNB per capita; $y=$ Taxa de crescimento do PIB; $E$ = Taxa de alfabetização; $M=$ Taxa de matrículas na escola secundária; $P=$ Taxa de crescimento populacional; $A=$ Participação da agricultura no PIB; $U$ = Participação da população do meio urbano; $S=$ Variável dummy para países socialistas.

Os resultados verificados por meio da aplicação do modelo (1a) podem ser resumidos da seguinte forma: 1) Foram encontradas evidências de exclusão do acesso à educação escolar por parte dos grupos de renda mais baixa. Contudo, melhorias no bem-estar foram identificadas, decorrentes de incrementos nas parcelas de renda dos segmentos mais pobres e de renda média da população, à custa dos mais ricos; 2) A taxa de crescimento da população demonstrou-se uma relevante variável explicativa em todas as equações, atuando de forma a desfavorecer as parcelas de renda dos grupos de renda baixa e média e a 
elevar a participação do grupo dos 20\% mais ricos; 3) O fato da participação da agricultura no PIB declinar a medida que o desenvolvimento ocorra induz a um processo de transferência de renda dos grupos de renda média para os de renda mais elevada. No entanto, o desenvolvimento também induz, de modo paralelo, a um deslocamento populacional em direção aos setores modernos (urbanos), sendo este processo favorecedor dos grupos de renda mais baixa à custa do rico; 4) Foi constatado que os países socialistas exibem uma estrutura de renda mais equitativa que os demais; 5) Foi encontrado suporte para as afirmações de aumento da desigualdade relativa nos estágios iniciais do desenvolvimento, porém os resultados não apoiaram a hipótese adicional da existência de uma redução absoluta dos níveis de renda nesta fase para a população mais pobre (Ahluwalia, 1976: 130-134).

No mesmo trabalho, Ahluwalia, utilizando a mesma divisão da população em participações na renda, procedeu à utilização do seguinte modelo reduzido, visando à captação das relações de longo prazo:

$$
L_{i}=\alpha+\beta_{1} Y+\beta_{2} Y^{2}+D-\varepsilon
$$

Onde: $\alpha=$ Intercepto; $L_{i}=$ Participação de cada grupo na renda; $Y=$ Logaritmo da renda per capita; $D=$ Variável dummy para países socialistas.

Segundo a equação (1b), para a existência de uma curva na forma de U-invertido, os coeficientes $\beta_{1}$ e $\beta_{2}$ devem assumir, respectivamente, os valores positivo e negativo. Nesse modelo, o autor assumia que as mudanças estruturais verificadas na aplicação do modelo mais geral (1a) estavam implícitas nas variáveis explicativas do modelo simples (1b). Com o intuito extra de testar a relação de curto prazo entre desigualdade e crescimento, Ahluwalia incluiu no modelo (1b) a Taxa de crescimento do PIB como uma variável explanatória adicional. $\mathrm{O}$ modelo adquiriu então a seguinte formulação:

$$
L_{i}=\alpha+\beta_{1} \mathrm{Y}+\beta_{2} \mathrm{Y}^{2}+\beta_{3} y+D+\varepsilon
$$

Onde: $\alpha$ = Intercepto; $L_{i}=$ Participação de cada grupo na renda; $Y=$ Logaritmo do PNB per capita; $y=$ Taxa de crescimento do PIB; $D=$ Variável dummy para países socialistas.

Foi encontrada uma relação estatisticamente significante entre a participação na renda dos vários grupos e o logaritmo do PNB per capita. Adicionalmente, e desde que uma relação significante fosse identificada quando apenas a variável PNB per capita fosse introduzida na forma quadrática, o autor revela ter encontrado indícios claros de que essa relação é não monotônica. Dessa forma, por meio dessa formulação, foi verificado o padrão de U-invertido na medida em que a participação de todos os grupos percentuais inicialmente declina e depois aumenta à medida que a renda per capita se eleva, exceto para o quintil mais rico, que segue o padrão oposto. A par deste resultado, foram 
encontradas evidências que negam a existência de uma relação sistemática de curto prazo entre o nível de desigualdade e a taxa de crescimento do PIB ao longo da década considerada (Ahluwalia, 1976: 129).

Outro estudo clássico sobre a curva de Kuznets, de caráter mais geral, foi realizado por Anand e Kanbur (1993 apud Jacinto e Tejada, 2004), onde os mesmos derivaram uma relação entre desigualdade e crescimento através da consideração do processo de mudança intersetorial da população implícito em Kuznets (1955) e da utilização de seis medidas de desigualdade. Em meio a essa derivação, os autores também estabelecem as condições necessárias à obtenção da curva no formato de U-invertido. Para a utilização do índice de $L$ de Theil, os autores propuseram o seguinte modelo:

$$
L=\alpha+\beta_{1} Y+\beta_{2} \log Y+\varepsilon
$$

Onde: $L=$ Índice de desigualdade $L$ de Theil; $Y=$ Renda per capita.

Nesse caso específico, a condição necessária para que as relações contidas na equação (1c) apresentem uma curva em forma de U-invertido é que os coeficientes $\beta_{1}$ e $\beta_{2}$ apresentem sinais negativo e positivo, respectivamente. As grandes contribuições desse estudo são a formalização da suposição de Kuznets e a recomendação por parte dos autores do uso de modelos específicos para os diversos índices de desigualdade. Anand e Kanbur (1993 apud Jacinto e Tejada) também aplicaram o modelo (1c) aos dados utilizados por Ahluwalia (1976) encontrando resultados opostos ao trabalho do mesmo, ou seja, a negação da curva em forma de U-invertido para a relação entre desigualdade e renda per capita, evidenciando a ausência de robustez nos resultados deste autor.

Os resultados distintos encontrados nos dois trabalhos mencionados acima ilustram o caráter controverso do conjunto de resultados presentes nos trabalhos que se propuseram a verificar empiricamente a existência da Curva de Kuznets. As controvérsias estão relacionadas à utilização das diversas medidas de desigualdade nas estimações e ao emprego de formas funcionais distintas. O quadro 1 a seguir expõe estudos adicionais sobre o tema, realizados por outros autores, explicitando o tipo e tamanho da amostra, o método empregado e as principais conclusões de cada trabalho. Interessante notar que a evolução dos métodos ocorre através de combinações de modelos alternativos e que tal combinação se apresenta como condição necessária ao teste da hipótese de Kuznets. 


\section{QUADRO 1. SUMÁRIO DOS ESTUDOS SOBRE A CURVA DE KUZNETS}

\begin{tabular}{|c|c|c|c|}
\hline Referência & $\begin{array}{c}\text { Âmbito do } \\
\text { estudo }\end{array}$ & Método & Conclusões \\
\hline $\begin{array}{l}\text { Ahluwalia } \\
\text { (1976) }\end{array}$ & $\begin{array}{l}\text { Amostra de } \\
60 \text { países: } 40 \\
\text { considerados } \\
\text { subdesenvolvi- } \\
\text { dos, } 6 \text { socialistas } \\
\text { e } 14 \text { desenvol- } \\
\text { vidos. }\end{array}$ & $\begin{array}{l}\text { Cross-section para desigualdade } \\
\text { em função da renda per capita } \\
\text { e uma dummy para países } \\
\text { socialistas. }\end{array}$ & $\begin{array}{l}\text { Os resultados apoiam a } \\
\text { hipótese do U invertido. }\end{array}$ \\
\hline $\begin{array}{l}\text { Braulke } \\
(1983)\end{array}$ & $\begin{array}{l}\text { Amostra de } 33 \text { pa- } \\
\text { íses com renda } \\
\text { homogênea. }\end{array}$ & $\begin{array}{l}\text { Modelo não linear para o Índice } \\
\text { de Gini na função de razões } \\
\text { setoriais de renda e população. }\end{array}$ & $\begin{array}{l}\text { Quando é considerada a } \\
\text { convergência entre as ren- } \\
\text { das setoriais o U invertido } \\
\text { apresenta uma redução em } \\
\text { sua fase inicial }\end{array}$ \\
\hline Ram (1989) & $\begin{array}{l}\text { Amostra com } 115 \\
\text { países para o } \\
\text { período } 1960-80\end{array}$ & $\begin{array}{l}\text { Cross-section para um modelo } \\
\text { quadrático do Indice de Theil em } \\
\text { função do PIB per capita. }\end{array}$ & $\begin{array}{l}\text { Os resultados apóiam a } \\
\text { hipótese do U invertido }\end{array}$ \\
\hline $\begin{array}{l}\text { Anand e } \\
\text { Kanbur } \\
(1993 b)\end{array}$ & $\begin{array}{l}\text { Amostra de } 60 \\
\text { países (dados de } \\
\text { Ahluwalia (1976) }\end{array}$ & $\begin{array}{l}\text { Cross-section para as funcionais } \\
\text { derivadas para } 6 \text { índices de } \\
\text { desigualdade em função do PIB } \\
\text { per capita. }\end{array}$ & $\begin{array}{l}\text { Os resultados não apoiam a } \\
\text { hipótese do U invertido. }\end{array}$ \\
\hline $\begin{array}{l}\text { Fields e } \\
\text { Jakubson } \\
(1994)\end{array}$ & $\begin{array}{l}\text { Amostra de } 20 \\
\text { países. }\end{array}$ & $\begin{array}{l}\text { Pooled cross-section e dados de } \\
\text { painel para o modelo quadrático } \\
\text { do índice de Gini como função da } \\
\text { renda per capita. }\end{array}$ & $\begin{array}{l}\text { Os resultados do pooled } \\
\text { cross-section apoiam a } \\
\text { existência do U invertido. } \\
\text { Porém, as estimações com } \\
\text { efeitos fixos contradizem } \\
\text { essa hipótese. }\end{array}$ \\
\hline $\begin{array}{l}\text { Hsing e Smyth } \\
\text { (1994) }\end{array}$ & $\begin{array}{l}\text { Séries de tempo } \\
\text { para economia } \\
\text { a mericana no } \\
\text { período } 1948-87 \text {. }\end{array}$ & $\begin{array}{l}\text { Estimação de um SUR para o } \\
\text { índice de Gini, considerando } \\
\text { separadamente as raças brancas } \\
\text { e negras. }\end{array}$ & $\begin{array}{l}\text { Os resultados apóiam a } \\
\text { hipótese do U invertido } \\
\text { para as duas raças e o } \\
\text { turning point coincide. }\end{array}$ \\
\hline $\begin{array}{l}\text { Dawson } \\
\text { (1997) }\end{array}$ & $\begin{array}{l}\text { Amostra dos } 20 \\
\text { países menos } \\
\text { desenvolvidos de } \\
\text { RAM (1989). }\end{array}$ & $\begin{array}{l}\text { Cross-section com modelos } \\
\text { quadráticos e semi log para o } \\
\text { coeficiente do Gini com respeito } \\
\text { à renda per capita. }\end{array}$ & $\begin{array}{l}\text { Os resultados apoiam a } \\
\text { hipótese do U invertido. }\end{array}$ \\
\hline $\begin{array}{l}\text { Deininger e } \\
\text { Squire (1998) }\end{array}$ & $\begin{array}{l}\text { Amostra de } 108 \\
\text { países para o } \\
\text { período } 1960-90 .\end{array}$ & $\begin{array}{l}\text { Cross-section para o crescimento } \\
\text { em função da renda, inverso da } \\
\text { desigualdade e educação. Dados } \\
\text { em painel do nível de desigualda- } \\
\text { de em função da renda média e do } \\
\text { sistema político do país. }\end{array}$ & $\begin{array}{l}\text { A desigualdade reduz o } \\
\text { crescimento econômico nos } \\
\text { países pobres, mas não nos } \\
\text { ricos. Os dados de séries de } \\
\text { tempo apóiam a hipótese } \\
\text { do U invertido. }\end{array}$ \\
\hline $\begin{array}{l}\text { Ogwang } \\
(2000)\end{array}$ & $\begin{array}{l}\text { A mostra de } \\
175 \text { países com } \\
\text { dados das Nações } \\
\text { Unidas para } 1994 .\end{array}$ & $\begin{array}{l}\text { Cross-section da desigualdade } \\
\text { entre países para PIB per capita e } \\
\text { vários índices de desenvolvimen- } \\
\text { to humano. }\end{array}$ & $\begin{array}{l}\text { A rela ção e n tre } \\
\text { desigualdade e PIB per } \\
\text { capita é maior do que com } \\
\text { relação aos índices de } \\
\text { desenvolvimento humano. }\end{array}$ \\
\hline $\begin{array}{l}\text { Sylvester } \\
(2000)\end{array}$ & $\begin{array}{l}\text { Amostra de } 54 \\
\text { países (dados de } \\
\text { Barro e Lee } \\
(1994))\end{array}$ & $\begin{array}{l}\text { Cross-section do crescimento em } \\
\text { função dos gastos em educação } \\
\text { e desigualdade de renda (índice } \\
\text { de Gini) }\end{array}$ & $\begin{array}{l}\text { Os gastos em educação } \\
\text { afetam o crescimento } \\
\text { no longo prazo e a } \\
\text { desigualdade condiciona o } \\
\text { crescimento (curto prazo). }\end{array}$ \\
\hline $\begin{array}{l}\text { Thornton } \\
(2001)\end{array}$ & $\begin{array}{l}\text { Amostra de } 96 \\
\text { países (dados de } \\
\text { Deininger e } \\
\text { Squire (1998)) }\end{array}$ & $\begin{array}{l}\text { Dados em painel do modelo } \\
\text { quadráticos da desigualdade em } \\
\text { função do PIB per capita }\end{array}$ & $\begin{array}{l}\text { Os resultados apoiam a } \\
\text { hipótese do U invertido }\end{array}$ \\
\hline
\end{tabular}

FONTE: Rodriguez e Menéndez, 2002 apud Jacinto e Tejeda (2004), modificado. 


\section{Metodologia}

Neste trabalho foram utilizados dados referentes ao Brasil de variáveis representativas do crescimento econômico e da desigualdade na distribuição de renda. Trata-se de séries dos índices de Gini e de Theil - $T$, e de Renda domiciliar per capita média ( $\mathrm{R} \$$ de 2002) para o período 1976-2007, totalizando uma série temporal de 32 observações. Tais dados foram obtidos através do sistema IPEADATA, disponibilizado pelo Instituto de Pesquisa Econômica Aplicada (IPEA).

O modelo econométrico utilizado no teste da curva de Kuznets, semelhante ao modelo simplificado utilizado por Ahluwalia (1976), foi o descrito abaixo:

$$
D=\beta_{1}+\beta_{2} X_{\mathrm{t}}+\beta_{3} X^{2}{ }_{t}+f_{\mathrm{t}}
$$

Onde: $D=$ Índice de Desigualdade (Gini ou Theil-T), $X=$ Renda per capita, $\beta_{1}=$ Intercepto e $t=$ Período de tempo (ano).

A restrição da equação (3a) para que a hipótese de Kuznets não seja refutada é a de que os coeficientes $\beta_{2}$ e $\beta_{3}$ devem assumir, respectivamente, valores' positivos e negativos $\left(\beta_{2}>\right.$ o e $\left.\beta_{3}<0\right)$.

Ademais, foi inicialmente suposto que os termos de erros $\left(\varepsilon_{t}\right)$ são variáveis aleatórias não correlacionadas com média zero e variância constante $\sigma^{2}$. Formalmente:

$$
E\left(\varepsilon_{\mathrm{t}}\right)=0, \operatorname{var}\left(\varepsilon_{\mathrm{t}}\right)=\sigma^{2} \mathrm{e} \operatorname{cov}\left(\varepsilon_{t}, \varepsilon_{s}\right)=0, \text { para } t \neq s .
$$

Entretanto, apesar das vantagens da disposição de dados em séries de tempo para o atendimento de parte dos objetivos deste estudo, o tratamento de dados de séries temporais frequentemente é acompanhado, de forma implícita, de determinados problemas característicos que podem vir a distorcer a eficiência dos estimadores e, por consequência, a qualidade do modelo em termos de sua capacidade explicativa, exigindo atenção do pesquisador quanto à deteç̧ão e emprego de métodos de superação dessas distorções. O mais comum desses problemas é o da presença de autocorrelação, que consiste na influência remanescente dos efeitos de um termo de erro sobre seu(s) sucessor(es) ou sobre os valores futuros de outras variáveis aleatórias. Isso decorre da característica de ordenamento natural dos dados de séries temporais ao longo do tempo, referentes ao comportamento de uma mesma unidade amostral ou de uma mesma variável. Em termos deste trabalho, o problema da autocorrelação pode ser interpretado sob a forma de um ajustamento na distribuição de renda decorrente de um choque econômico, por exemplo, o que afeta a estrutura distributiva durante mais de um período sucessivo de tempo através de um efeito cumulativo. 
Tecnicamente, na presença de autocorrelação os termos de erro passam a ser correlacionados, de forma que:

$$
\operatorname{cov}\left(\varepsilon_{t}, \varepsilon_{s}\right) \neq 0, \text { para } t \neq s .
$$

Num primeiro momento, o método empregado para detectar a autocorrelação foi o teste dos limites de Durbin-Watson. Neste, dado o modelo empregado no presente trabalho (2a), o termo de erro passa a assumir a forma auto-regressiva de primeira ordem,

$$
\varepsilon_{t}=\rho \varepsilon_{t-1}+v_{t}
$$

Onde: $\rho=$ coeficiente de correlação e $v_{t}=$ termo composto de erros independentes e aleatórios.

Importante notar que, caso $\rho=0$, $\varepsilon_{t}$ será igual a $v_{t}$, de modo que os erros (3b) serão não autocorrelacionados. Dessa forma, o método estabelece a hipótese nula de que não há autocorrelação $\left(\mathrm{H}_{0}: \rho=0\right)$, contra a hipótese alternativa bilateral de existência de autocorrelação $\left(\mathrm{H}_{1}: \rho \neq 0\right)$. Calculando-se o valor estimado do coeficiente de correlação $\rho$, é possível testar em seguida se tal estimativa é significativamente maior que zero. Entretanto, dadas as dificuldades de se obter a distribuição de probabilidade exata de $\rho$, o presente método calcula um indicador distinto, cuja distribuição de probabilidade é passível de ser calculada. Este indicador é conhecido como estatística $d$ de Durbin-Watson, que está estreitamente relacionado ao $\rho$, de forma que: $\mathrm{d} \approx 2(1-\rho)$.

Isso implica que, se $\rho=0$ (ausência de autocorrelação), então a estatística de Durbin-Watson será d $\approx 2$. E, ainda, caso $\rho=1$ (presença de autocorrelação), a estatística tomará o valor de $\mathrm{d} \approx 0$. Portanto, se o valor de $d$ estiver "suficientemente próximo" de 2, isto será uma indicação de que os erros do modelo não são autocorrelacionados. Por outro lado, se o valor de $d$ for "suficientemente próximo" de o, isso indicará que os erros do modelo são autocorrelacionados. Para se determinar que valores são aceitos como "suficientemente próximos" a o ou a 2, é necessário observar os valores críticos da estatística $d$, que dependem do número de observações " $T$ " e do número de parâmetros " $K$ ". Esses valores críticos são os chamados de limite inferior $\left(d_{L}\right)$ e limite superior $\left(d_{v}\right)$. De posse destes valores, deve-se obedecer às seguintes regras de decisão, as quais constituem o teste dos limites dêDurbin-Watson em sua eŝsência:

Se $\mathrm{o}<d<d_{L}$, deve-se rejeitar $\mathrm{H}_{\mathrm{o}}: \rho=0$;

Se $d_{L}<d<d_{U}$, o teste é inconclusivo quanto a $\mathrm{H}_{\mathrm{o}}: \rho=0$;

Se $d_{U}<d<4-d_{U}$, não rejeitar $\mathrm{H}_{\mathrm{o}}: \rho=0$; 
Se $4-d_{U} \leq d \leq 4-d_{L}$, o teste é inconclusivo quanto a $\mathrm{H}_{\mathrm{o}}: \rho=0$;

Se $4-d_{L}<d<4$, deve-se rejeitar $\mathrm{H}_{0}: \rho=0$.

A existência de um intervalo que não atribui uma conclusão ao teste é, sem dúvida, uma limitação do teste. No entanto, caso em algum momento seja verificado que o valor da estatística $d$ se encontra entre o limite inferior e superior $\left(d_{L}\right.$ e $\left.d_{U}\right)$, pode-se recorrer a outro teste, conhecido por Teste dos Multiplicadores de Lagrange (teste LM) ou Teste Breusch-Godfrey (teste BG).

Para executar o teste LM é necessário antes modificar o modelo (2a), incluindo a equação (2b) no mesmo, tornando-o um modelo autoregressivo de primeira ordem $\operatorname{AR}(1)$ :

$$
D=\beta_{1}+\beta_{2} X_{t}+\beta_{3} X_{t}^{2}+\mathrm{t}_{1} f_{t} \div \mathbf{1}+
$$

Onde a hipótese nula a ser testada é $\mathrm{H}_{0}: \rho_{1}=0$, isto é, não há correlação serial de primeira ordem. Especificada a hipótese nula, deve-se estimar (2c) pelo método dos Mínimos Quadrados Ordinários (MQO) a fim de se obter os resíduos $\varepsilon_{\mathrm{t}}$. Em seguida, efetua-se a regressão de $\varepsilon_{\mathrm{t}}$ contra as variáveis incluídas no modelo, considerando os valores defasados dos resíduos estimados na etapa anterior. Dessa forma, efetua-se a seguinte regressão objetivando obter seu $\mathrm{R}^{2}$ :

$$
f=\alpha+\beta_{1} X_{t}+\beta_{1} X_{t}^{2}+t_{1} f_{\mathrm{t}-1}+y_{\mathrm{t}}
$$

À medida que o tamanho da amostra tende ao infinito, vezes o valor do $\mathrm{R}^{2}$ obtido na regressão (2d) tende assintoticamente à distribuição qui-quadrado com $p$ graus de liberdade. Formalmente, $(n-p) \mathrm{R}^{2} \sim \chi^{2}$. Caso o valor de $(n-p) \mathrm{R}^{2}$ exceda o valor crítico de qui-quadrado no nível de significância especificado (nesse caso 5\%), a hipótese nula é rejeitada, pois, nesse caso, $\rho \neq 0$. Alternativamente, pode-se observar o valor- $p$ do teste BG, verificando se o mesmo ultrapassa o nível de significância pré-estabelecido, o que ocasionaria a não rejeição de $\mathrm{H}_{\mathrm{o}}$.

Uma vez detectada a autocorrelação, é necessário aplicar métodos de correção. Quando necessário, o método utilizado no presente estudo foi o dos Erros Auto-Regressivos de Primeira Ordem “AR(1)”, já mencionado anteriormente, que consiste numa transformaçãô do modelo original, o qual passa a considerar a existência de correlação serial entre os erros que, por sua vez, passa a ser estimado pelo método dos Mínimos Quadrados Generalizados. 


\section{Análise de resultados}

\subsection{Aplicações da curva de Kuznets ao Brasil}

Inicialmente, foi utilizado o Índice de Theil $\mathrm{T}$ como indicador de desigualdade. O tratamento do modelo foi efetuado por meio do uso do Software Econometric Views (Eviews). Os resultados estimados pelo Método dos Mínimos Quadrados Ordinários (MQO) são apresentados na Tabela 1 abaixo:

TABELA 1 . RELAÇÃO ENTRE ÍNDICE T DE THEIL E RENDA PER CAPITA (X)

\begin{tabular}{l|cc|c|c}
\hline \multicolumn{5}{c}{ Variável Dependente: Theil T } \\
\hline \multicolumn{1}{c}{ Variável } & Coeficiente & Erro Padrão & Est. $T$ & Prob. \\
\hline $\mathrm{C}$ & 0.266410 & 0.492715 & 0.540697 & 0.5928 \\
$\mathrm{X}$ & 0.003216 & 0.003079 & 1.044 .363 & 0.3049 \\
$\mathrm{X}^{\wedge} 2$ & $-5.43 \mathrm{E}-06$ & $4.76 \mathrm{E}-06$ & -1.139 .431 & 0.2638 \\
\hline $\mathrm{R}^{2}$ Ajustado & 0.014429 & & & \\
Est. Durbin-Watson & 0.793623 & & & \\
\hline
\end{tabular}

Nota-se que os coeficientes, embora apresentem os sinais esperados $\left(\beta_{2}>0\right.$ e $\beta_{3}<0$ ), estão muito aquém de serem estatisticamente significativos ao nível de confiança de $95 \%$. É perceptível também o reduzido poder de explicação (inferior a 2\%) do modelo na explicação do nível de desigualdade medido pelo índice $\mathrm{T}$ de Theil. Entretanto, ao submeter este resultado ao teste dos limites de Durbin-Watson, a fim de verificar a presença de autocorrelação nos termos de erro, verifica-se a presença de correlação serial positiva nos mesmos, visto que $0<d<d_{L}$, onde $d=1,309$ para o número de observações e parâmetros utilizados nesse modelo. Visando à correção deste problema, foi empregado o método dos erros auto regressivos de primeira ordem, AR(1). Os resultados da aplicação do método de mínimos quadrados ordinários após a transformação do modelo pelo método AR(1) foram os seguintes:

TABELA 2. ÍNDICE T DE THEIL E RENDA PER CAPITA APÓS AR(1)

\begin{tabular}{l|c|c|c|c}
\hline \multicolumn{5}{c}{ Variável Dependente: Theil T } \\
\hline \multicolumn{1}{c}{ Variável } & Coeficiente & Erro Padrão & Est. $T$ & Prob. \\
\hline $\mathrm{C}$ & -0.240004 & 0.320478 & -0.748894 & 0.4604 \\
$\mathrm{X}$ & 0.005401 & 0.001919 & 2.813 .794 & 0.0090 \\
$\mathrm{X}^{\wedge} 2$ & $-7.57 \mathrm{E}-06$ & $2.86 \mathrm{E}-\mathrm{o6}$ & -2.646 .966 & 0.0134 \\
$\mathrm{AR}(1)$ & 0.749809 & 0.112727 & 6.651 .528 & 0.0000 \\
\hline $\mathrm{R}^{2}$ Ajustado & 0.523565 & & & \\
Est. Durbin-Watson & 1.716341 & & & \\
& 0,541456 & & & 0.461830 \\
Est. Breusch-Godfrey & & & &
\end{tabular}


De posse do novo valor da estatística $d$ e procedendo-se novamente ao teste dos limites, verifica-se agora que a hipótese nula de ausência de autocorrelação não pode ser rejeitada, visto que $d_{U}<d<4-d_{U}$, onde $d=1,574$ para trinta $\mathrm{e}$ duas observações e duas variáveis explanatórias. O mesmo resultado é atestado mediante o uso do teste Breusch-Godfrey, cuja probabilidade, de 0,461 , resulta na não rejeição de $\mathrm{H}_{0}$. Ademais, pode-se observar que os coeficientes $\beta_{2}$ e $\beta_{3}$ continuam a apresentar os sinais esperados para a obtenção do padrão de U-invertido entre desigualdade e renda per capita, ou seja, $\beta_{2}$ positivo e $\beta_{3}$ negativo. Os coeficientes também passaram a ser estatisticamente significantes ao nível de confiança de 95\%, a exceção do intercepto. Vale notar que o modelo, após a introdução do AR(1), explica em aproximadamente 52,36\% a interação entre desigualdade e renda per capita, um resultado muito superior aos diversos trabalhos realizados com dados de regiões e municípios brasileiros que, embora também confirmem a relação indicada pela Curva de Kuznets, apontam um poder explicativo bem inferior ao encontrado no presente exercício econométrico. ${ }^{6}$

Os resultados da estimação da relação entre desigualdade e renda per capita refletidos na forma da curva de Kuznets estimada podem ser visualizados por meio do gráfico 1 abaixo. Os mesmos sugerem que, em média, à medida que a renda per capita aumenta, a desigualdade se move na mesma direção até um turning-point, estimado em 0,743 Theil $\mathrm{T}$, a partir do qual adquire uma tendência progressiva de redução com o aumento da renda.

O nível de desigualdade do turning-point estimado está associado a uma renda per capita de $\mathrm{R} \$ 296,43$.

GRÁFICO 1. CURVA DE KUZNETS ESTIMADA - THEIL T (1976-2007)

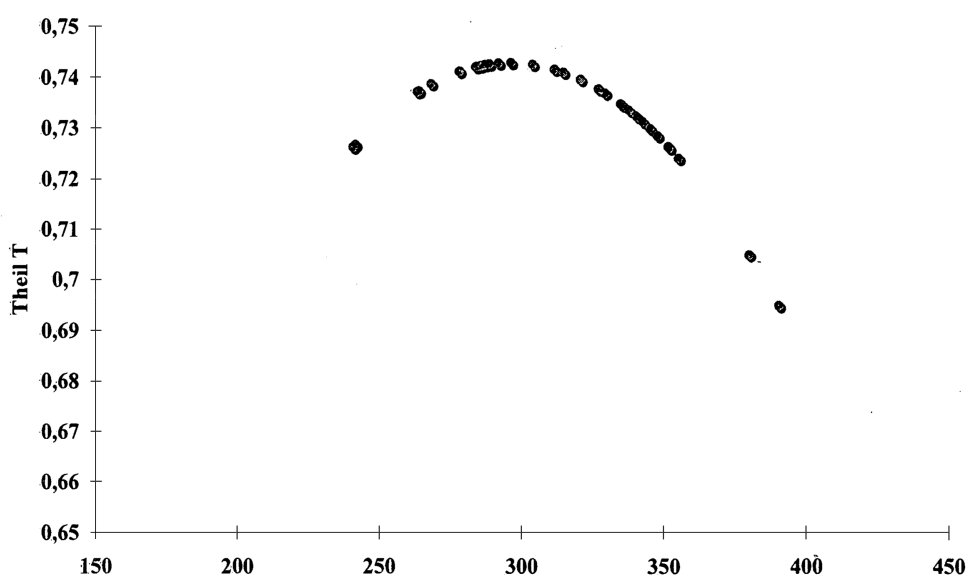

FONTE: elaboração dos autores.

6 Ver, por exemplo, Jacinto e Tejada (2004) e Salvato et al (2006). 
Como forma de obter uma indicação adicional para a validade da análise feita acima, o mesmo exercício foi feito para o Índice de Gini como variável dependente. Os resultados para o modelo não autorregressivo são descritos abaixo:

TABELA 3 . RELAÇÃO ENTRE ÍNDICE DE GINI E RENDA PER CAPITA (X)

\begin{tabular}{l|cccc|c}
\hline \multicolumn{5}{c}{ Variável Dependente: Gini } \\
\hline \multicolumn{1}{c}{ Variável } & Coeficiente & Erro Padrão & Estat. T & Prob. \\
\hline $\mathrm{C}$ & 0.441325 & 0.129000 & 3421116 & 0.0019 \\
$\mathrm{X}$ & 0.001088 & 0.000806 & 1.349 .570 & 0.1876 \\
$\mathrm{X}^{\wedge} 2$ & $-1.87 \mathrm{E}-06$ & $1.25 \mathrm{E}-06$ & -1.496 .896 & 0.1452 \\
\hline $\mathrm{R}^{2}$ Ajustado & 0.089676 & & & \\
Estat. Durbin-Watson & 0.573977 & & & \\
\hline
\end{tabular}

Aqui, os sinais dos coeficientes $\left(\beta_{2}>\right.$ o e $\beta_{3}<0$ ) também são condizentes com a teoria do U-invertido. Porém, ambos os parâmetros foram rejeitados ao nível de significância de $5 \%$ e o indicador $\mathrm{R}^{2}$, que mede o poder explicativo das variáveis independentes explícitas no modelo sobre a variável dependente, apresentou um nível demasiadamente baixo. Contudo, o baixo valor obtido na estatística $d$ de Durbin-Watson atesta a presença de autocorrelação, visto que seu valor, 0,573 , é inferior ao $d_{L}$ crítico de 1,309.

Aplicando o modelo autorregressivo de primeira ordem AR(1) aos mesmos dados de Índice de Gini e Renda per capita utilizados acima, é possível observar os seguintes resultados:

TABELA 4 . ÍNDICE DE GINI E RENDA PER CAPITA (X) APÓS AR(1)

\begin{tabular}{l|cccc}
\hline \multicolumn{5}{c}{ Variável Dependente: Gini } \\
\hline $\mathrm{C}$ & Coeficiente & Erro Padrão & Estat. T & Prob. \\
\hline $\mathrm{X}$ & 0.394459 & 0.073899 & 5.337 .805 & 0.0000 \\
$\mathrm{X}^{\wedge} 2$ & 0.001126 & 0.000438 & 2.571 .267 & 0.0160 \\
$\mathrm{AR}(1)$ & $-1.67 \mathrm{E}-06$ & $6.52 \mathrm{E}-07$ & -2.560 .044 & 0.0164 \\
\hline $\mathrm{R}^{2}$ Ajustado & 0.855429 & 0.111700 & 7.658 .262 & 0.0000 \\
Est. Durbin-Watson & 0.660918 & & & \\
Est. Breusch-Godfrey & 1.517 .091 & & & \\
\hline
\end{tabular}

A primeira conclusão, ao se observar o novo valor apresentado pela estatística $d$, é de que a mesma encontra-se na região inconclusiva quanto à correção da autocorrelação, visto que $d_{L}=1,309 \leq d=1,517 \leq d_{U}=1,574$, ou 'seja, o teste não é capaz de inferir se o problema da correlação serial foi solucionado. Nessa situação, cabe a comprovação da eficiência do esquema AR(1) mediante o teste BG. O mesmo apresentou uma probabilidade de 0,134 , muito acima do nível de significância de $5 \%$, o que nos leva a concluir pela não rejeição de $\mathrm{H}_{\mathrm{o}}$. 
Dados os testes efetuados até o momento, pode-se então observar que os coeficientes do modelo apresentam sinais $\left(\beta_{2}>\right.$ o e $\left.\beta_{3}<0\right)$ que confirmam a hipótese de Kuznets de que a desigualdade na distribuição de renda aumenta nos estágios iniciais do crescimento econômico, adquirindo uma tendência decrescente num momento posterior, logo após atingir um turningpoint. Todos os coeficientes, incluindo o intercepto, são estatisticamente significativos ao nível de $5 \%$. Além disso, o $\mathrm{R}^{2}$ da ordem de aproximadamente $66 \%$ revela que a capacidade explicativa do modelo apresenta um nível bastante satisfatório.

Este resultado é uma evidência significativa da aplicabilidade da hipótese de Kuznets em explicar o comportamento padrão da relação entre desigualdade na distribuição de renda e crescimento econômico de longo prazo no Brasil. Segundo o referido modelo, em média, à medida que ocorre o crescimento econômico (evidenciado pelo aumento da renda per capita) o grau de desigualdade na distribuição da renda (representado pelo índice de Gini) sofre elevações iniciais, atingindo um ponto máximo estimado em cerca de o,60o Gini, passando a sofrer reduções sucessivas a partir do mesmo no decorrer do processo de crescimento da renda per capita. O nível de renda per capita associado ao nível de desigualdade estimado no ponto de inflexão é de aproximadamente $\mathrm{R} \$ 292,10$.

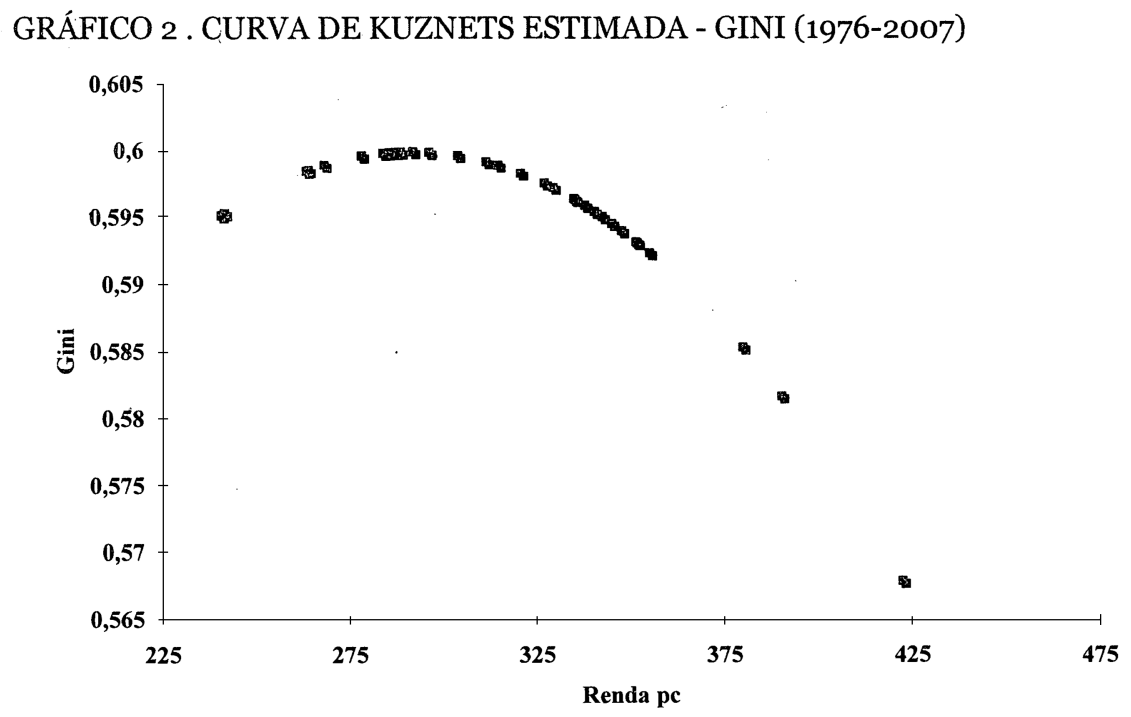

FONTE: elaboração dos autores. 
O resultado encontrado para o índice de Gini, ainda mais significativo que o verificado quando se utilizou o índice de Theil T, ganha ainda mais relevância pelo fato de o padrão de U invertido ter sido testado e verificado para ambos os índices de desigualdade. Desse modo, esse exercício com proxy's distintas da desigualdade confere maior segurança a ambos os resultados.

\subsection{Empobrecimento absoluto e relativo: comparações ao longo do tempo}

A fim de verificar a hipótese mais forte acerca da Teoria do U-invertido, a saber, a de que a elevação da desigualdade nos períodos iniciais é acompanhada de uma redução absoluta nos níveis de renda dos grupos mais pobres da população, foi feita a seguir uma comparação entre a estrutura da distribuição de renda no Brasil nos anos de 1981, 1989, 1999 e 2007. Tal comparação também é útil para visualizar as mudanças de longo prazo na referida estrutura e para delimitar intervalos no tempo, onde comportamentos específicos (diferenciados) da dinâmica distributiva se verificam.

As Tabelas 5 a 9 situadas abaixo apresentam informações relativas à renda média, participação na renda total e renda relativa (em relação à renda média) para diversos grupos percentuais da população brasileira nos anos acima mencionados. Além disso, a Tabela 9 também mostra os valores dos índices de concentração e desigualdade (Gini, Theil T e a razão entre a renda média obtida pelo grupo dos $10 \%$ mais ricos em relação à auferida dos $40 \%$ mais pobres) para o país em cada um desses anọs.

Nas tabelas a seguir, os decis da população estão organizados do menor ao maior nível de renda.

TABELA 5. Participação na Renda Total - em decis (1981/1989/1999/2007)

\begin{tabular}{|l|c|c|c|c|c|c|c|}
\hline \multirow{2}{*}{ Percentil } & \multicolumn{7}{|c|}{ Percentagem da renda } \\
\cline { 2 - 8 } & 1981 & 1989 & 1999 & 2007 & $\begin{array}{c}1981 / 89 \\
\text { (em \%) }\end{array}$ & $\begin{array}{c}1989 / 99 \\
\text { (em \%) }\end{array}$ & $\begin{array}{c}1999 / 07 \\
\text { (em \%) }\end{array}$ \\
\hline $10-$ & 0,88 & 0,64 & 0,74 & 0,88 & $-26,72$ & 14,79 & 18,69 \\
\hline 10 & 1,78 & 1,37 & 1,67 & 2,02 & $-23,00$ & 21,65 & 21,10 \\
\hline 10 & 2,56 & 2,04 & 2,47 & 2,91 & $-20,61$ & 21,26 & 17,95 \\
\hline 10 & 3,44 & 2,82 & 3,36 & 3,89 & $-18,16$ & 19,51 & 15,49 \\
\hline 10 & 4,48 & 3,76 & 4,45 & 5,05 & $-16,05$ & 18,49 & 13,28 \\
\hline 10 & 5,81 & 5,06 & 5,70 & 6,48 & $-12,91$ & 12,66 & 13,66 \\
\hline 10 & 7,68 & 6,85 & 7,53 & 8,11 & $-10,73$ & 9,87 & 7,76 \\
\hline 10 & 10,59 & 9,85 & 10,46 & 10,81 & $-6,93$ & 6,14 & 3,38 \\
\hline 10 & 16,38 & 16,11 & 16,35 & 16,02 & $-1,65$ & 1,48 & $-2,00$ \\
\hline $10+$ & 46,40 & 51,50 & 47,27 & 43,83 & 10,97 & $-8,22$ & $-7,27$ \\
\hline
\end{tabular}

FONTE: Elaboração dos autores, dados do IPEA. 
SANTOS, W. O.; MOURA, F. R.; SILVA, A.R.S.; MATOS, D. L.; FARIAS, T. A. A teoria do U invertido...

TABELA 6. Renda per capita Média (R\$ de 2002) - em decis (1981/1989/1999/2007)

\begin{tabular}{|l|r|r|r|r|r|r|r|}
\hline \multirow{2}{*}{ Percentil } & \multicolumn{7}{|c|}{ Renda per capita Média } \\
\cline { 2 - 8 } & \multicolumn{1}{|c|}{1981} & 1989 & 1999 & 2007 & $\begin{array}{c}1981 / 89 \\
(\mathrm{em} \%)\end{array}$ & $\begin{array}{c}1989 / 99 \\
(\mathrm{em} \%)\end{array}$ & $\begin{array}{c}1999 / \text { o7 } \\
(\mathrm{em} \%)\end{array}$ \\
\hline $10-$ & 24,43 & 21,55 & 24,76 & 34,21 & $-11,81$ & 14,90 & 38,16 \\
\hline 10 & 49,56 & 45,92 & 55,92 & 78,82 & $-7,33$ & 21,77 & 40,96 \\
\hline 10 & 71,39 & 68,21 & 82,80 & 113,68 & $-4,45$ & 21,38 & 37,30 \\
\hline 10 & 95,77 & 94,34 & 112,85 & 151,71 & $-1,50$ & 19,63 & 34,44 \\
\hline 10 & 124,65 & 125,95 & 149,39 & 196,97 & 1,04 & 18,61 & 31,86 \\
\hline 10 & 161,83 & 169,62 & 191,28 & 253,07 & 4,81 & 12,77 & 32,30 \\
\hline 10 & 213,69 & 229,59 & 252,50 & 316,72 & 7,44 & 9,98 & 25,43 \\
\hline 10 & 294,78 & 330,18 & 350,78 & 422,12 & 12,01 & 6,24 & 20,34 \\
\hline 10 & 456,02 & 539,78 & 548,33 & 625,46 & 18,37 & 1,58 & 14,07 \\
\hline $10+$ & $1.291,91$ & $1.725,43$ & $1.585,23$ & $1.711,13$ & 33,56 & $-8,13$ & 7,94 \\
\hline
\end{tabular}

FONTE: Elaboração dos autores, dados do IPEA.

TABELA 7. Renda Relativa (em relação à Renda Média) - em decis (1981/1989/1999/2007)

\begin{tabular}{|l|c|c|c|c|c|c|c|}
\hline \multirow{2}{*}{ Percentil } & \multicolumn{7}{|c|}{ Renda Relativa } \\
\cline { 2 - 8 } & 1981 & 1989 & 1999 & 2007 & $\begin{array}{c}1981 / 89 \\
(\mathrm{em} \mathrm{\% )}\end{array}$ & $\begin{array}{c}1989 / 99 \\
(\mathrm{em} \%)\end{array}$ & $\begin{array}{c}1999 / 07 \\
(\mathrm{em} \%)\end{array}$ \\
\hline $10-$ & 0,09 & 0,06 & 0,07 & 0,09 & $-26,72$ & 14,79 & 18,69 \\
\hline 10 & 0,18 & 0,14 & 0,17 & 0,20 & $-23,00$ & 21,65 & 21,10 \\
\hline 10 & 0,26 & 0,20 & 0,25 & 0,29 & $-20,61$ & 21,26 & 17,95 \\
\hline 10 & 0,34 & 0,28 & 0,34 & 0,39 & $-18,16$ & 19,51 & 15,49 \\
\hline 10 & 0,45 & 0,38 & 0,45 & 0,50 & $-16,05$ & 18,49 & 13,28 \\
\hline 10 & 0,58 & 0,51 & 0,57 & 0,65 & $-12,91$ & 12,66 & 13,66 \\
\hline 10 & 0,77 & 0,69 & 0,75 & 0,81 & $-10,73$ & 9,87 & 7,76 \\
\hline 10 & 1,06 & 0,99 & 1,05 & 1,08 & $-6,93$ & 6,14 & 3,38 \\
\hline 10 & 1,64 & 1,61 & 1,63 & 1,60 & $-1,65$ & 1,48 & $-2,00$ \\
\hline $10+$ & 4,64 & 5,15 & 4,73 & 4,38 & 10,97 & $-8,22$ & $-7,27$ \\
\hline
\end{tabular}

FONTE: Elaboração dos autores, dados do IPEA.

TABELA 8. Participação na Renda Total - Percentis (1981/1989/1999/2007)

\begin{tabular}{|c|c|c|c|c|c|c|c|c|}
\hline Ano & $1+$ & $10+$ & $50-$ & $20-$ & $\begin{array}{c}\text { Variação 1 } \\
\text { (em \%) }\end{array}$ & $\begin{array}{c}\text { Variação 10 + } \\
\text { (em \%) }\end{array}$ & $\begin{array}{c}\text { Variação 50 - } \\
\text { (em \%) }\end{array}$ & $\begin{array}{c}\text { Variação 20 - } \\
\text { (em \%) }\end{array}$ \\
\hline 1981 & 12,67 & 46,40 & 13,14 & 2,66 & - & - & - & - \\
\hline 1989 & 16,48 & 51,50 & 10,62 & 2,01 & 30,05 & 10,97 & $-19,14$ & $-24,23$ \\
\hline 1999 & 13,24 & 47,27 & 12,69 & 2,41 & $-19,67$ & $-8,22$ & 19,48 & 19,46 \\
\hline 2007 & 12,48 & 43,83 & 14,74 & 2,90 & $-5,71$ & $-7,27$ & 16,12 & 20,36 \\
\hline
\end{tabular}

FONTE: Elaboração dos autores, dados do IPEA.

TABELA 9: Índices Agregados (1981/1989/1999/2007)

\begin{tabular}{|c|c|c|c|c|c|c|c|c|}
\hline Ano & $\begin{array}{c}\text { Renda pc } \\
\text { média }\end{array}$ & $\begin{array}{c}\text { Variação } \\
\text { Renda média } \\
(\%)\end{array}$ & Gini & $\begin{array}{c}\text { Variação } \\
\text { Gini (\%) }\end{array}$ & Theil T & $\begin{array}{c}\text { Variação } \\
\text { Theil T (\%) }\end{array}$ & $\begin{array}{c}10+/ \\
40-\end{array}$ & $\begin{array}{c}\text { Variação } \\
10+/ / 40- \\
(\%)\end{array}$ \\
\hline 1981 & 278,40 & - & 0,584 & - & 0,682 & - & 21,43 & - \\
\hline 1989 & 335,06 & 20,35 & 0,636 & 8,79 & 0,889 & 30,36 & 30,00 & 40,02 \\
\hline 1999 & 335,38 & 0,10 & 0,594 & $-6,54$ & 0,711 & $-20,00$ & 22,95 & $-23,52$ \\
\hline 2007 & 390,39 & 16,40 & 0,556 & $-6,44$ & 0,623 & $-12,35$ & 18,09 & $-21,18$ \\
\hline
\end{tabular}

FONTE: Elaboração dos autores, dados do IPEA. *Em R\$ de 2002. 
Começando pelo período 1981-1989, é possível perceber com nitidez um movimento de transferência de renda da população mais pobre para a mais rica. $\mathrm{O}$ fato agravante é que isso também ocorre em termos absolutos.

Conforme ilustra a Tabela 6, são observados ganhos de renda a partir do quinto decil, em especial para os três decis da população com renda mais alta, à custa de reduções absolutas na renda média dos quatro decis mais pobres da população, indicando redução do nível de bem-estar no período. Esse movimento beneficiou proporcionalmente mais o grupo dos $10 \%$ mais ricos, que ao final da década de 1980 possuía uma renda cinco vezes superior à renda média do conjunto da população, como mostra a Tabela 7.

Tal favorecimento fica ainda explícito na Tabela 5, que mostra reduções contínuas e decrescentes (sucessivamente menores para os mais ricos) da participação de todos os decis na renda total, com exceção do decil mais rico, que aumenta em aproximadamente $11 \%$ sua participação. A redução da participação na renda total foi maior para o grupo dos $10 \%$ mais pobres (-26,7\%), o qual, após se situar no segundo pior nível de participação relativa de toda a série, demoraria algo em torno de 15 anos para retornar a níveis próximos da participação que detinha em 1981 (o,88\% da renda total), fechando a série (a despeito dos ganhos proporcionais individuais dos períodos 1989/99 e 1999/07, que serão comentados mais adiante) com uma renda domiciliar per capita média de míseros $\mathrm{R} \$ 34,21$.

Os índices agregados de desigualdade corroboram o aumento da concentração verificada na observação dos decis, através da elevação dos índices de Gini em $8,79 \%$ e de Theil T em 30,36\%. Outro indicador que dá uma ideia bastante razoável do aumento da inequidade neste período é a razão entre a renda média auferida pelos $10 \%$ mais ricos em relação à auferida pelos $40 \%$ mais pobres (10+/40-). Ao longo do período, o valor deste indicador aumentou de 21,4 para 30 (+ 40\%). Assim, em 1989, o grupo dos $10 \%$ mais ricos da população auferia uma renda média 30 vezes superior àquela auferida pelo grupo dos 40\% mais pobres (ver Tabela 9).

Diante disso, dadas as evidências encontradas nas estimações realizadas na subseção anterior de que a desigualdade de renda no Brasil obedece ao padrão médio especulado por Kuznets, no qual a desigualdade sofre incrementos sucessivos nos períodos iniciais do crescimento até um turning-point, decrescendo nos estágios posteriores, as informações acima constituem um forte apoio para a hipótese mais forte de que o aumento da desigualdade nos períodos iniciais é acompanhado de reduções absolutas na renda média dos grupos de renda mais baixa. Dito de outro modo, foram encontradas evidências de que o crescimento econômico dos estágios iniciais do desenvolvimento possui caráter "antipobre", no sentido de beneficiar proporcionalmente mais a parcela mais rica da população em detrimento dos grupos de renda mais baixa, com o prejuízo adicional da redução em termos absolutos da renda média destes últimos. 
Movimento oposto à concentração acima mencionada foi o de redução da inequidade verificada no período 1989-1999. Neste, percebe-se um aumento da participação no total da renda em todos os decis da população em detrimento unicamente do decil mais rico, que apresentou redução de $8,22 \%$ em sua participação (Tabela 5). Além disto, a redução da participação foi ainda mais elevada no percentil mais rico da população, $-19,67 \%$, proporção similar aos ganhos da metade mais pobre da população e do grupo dos $20 \%$ que detêm os níveis mais baixos de renda (Tabela 8).

Em 1989-1999, também ocorreu redução da desigualdade em termos absolutos. A Tabela 6 mostra ganhos na renda per capita média para todos os grupos da população, a exceção dos $10 \%$ mais ricos. Os maiores ganhos proporcionais foram registrados do segundo ao quinto decis de renda mais baixa.

Além disso, também aqui os índices mais agregados corroboram a redução da desigualdade, tendo em vista as reduções dos coeficientes de Gini e Theil $\mathrm{T}$ da ordem de $6,5 \%$ e $20 \%$, respectivamente, além da significativa redução de $23,5 \%$ da razão 10+/40-, conforme observado na Tabela 9.

Assim como no período 1981/89, a média da renda domiciliar per capita em 1999-2007 sofreu um acréscimo significativo $(+16,4 \%)$, conforme exposto na Tabela 9. Contudo, neste intervalo de tempo, ao contrário dos demais períodos, todos os grupos da população foram beneficiados por acréscimos absolutos de renda, conforme ilustra a Tabela 6 . Em termos proporcionais, este aumento absoluto foi maior para os grupos de renda mais baixa, como os quatro decis mais pobres da população, que sofreram incrementos na renda média que variam de $34,5 \%$ até $41 \%$.

Em termos de participação na renda total, verifica-se uma dinâmica semelhante à verificada no período anterior, ou seja, ganhos de participação relativa de toda a população, a exceção do grupo de renda mais alta (Tabela 5). Contudo, há algumas diferenças entre estas variações e as registradas anteriormente.

A primeira é que, além dos 10\% mais ricos, o decil imediatamente anterior também registrou redução em sua participação, ainda que em menor grau $(-7,27 \%$ e $-2 \%$, respectivamente).

A segunda se refere ao fato de que os ganhos de participação relativa dos grupos economicamente menos favorecidos ocorreram porque os incrementos na renda per capita média dos mesmos foram proporcionalmente maiores que os ganhos dos grupos mais ricos, e não em decorrência da redução da renda média destes últimos, como no período anterior (ver Tabelas 5 e 6).

A terceira distinção é que neste período, ao contrário do anterior, a redução proporcional da participação dos $10 \%$ mais ricos foi maior que a do grupo correspondente ao percentil da população que detém o nível de renda mais elevado (ver Tabela 8). 
Outra diferença é o fato da dança distributiva do período 1989-1999 ter ocorrido num cenário de estagnação econômica, com a renda per capita média (afora variações cíclicas de curto prazo) permanecendo praticamente constante ao fim do período, ao passo que no período 1999-2007, a exemplo de 1981-1989, as mudanças distributivas ocorreram num ambiente de crescimento da renda média. Porém, ao inverso deste último, o período 19992007 beneficiou proporcionalmente mais as partes da população que detêm as rendam mais baixas, conforme perceptível nas Tabelas 5 e 9.

Ainda neste período, e assim como nos dois anteriores, os índices de Gini e de Theil $\mathrm{T}$ e a razão 10+/40- reafirmam o resultado da análise dos decis, no sentido de uma redução da desigualdade acima discutida.

Dessa forma, diante do exposto acima e das informações presentes nas Tabelas 5 a 9, é razoável afirmar que a estrutura distributiva já foi pior que a presente no ano de 2007. No entanto, especialmente se for feita a comparação entre os extremos da série (1981 e 2007), verificamos que, para um período tão longo de tempo, as modificações se configuram demasiadamente pífias. Em 2007, por exemplo, o decil mais pobre continua auferindo uma renda equivalente a apenas $9 \%$ da renda média para a totalidade da população, mesmo nível de renda relativa verificado 27 anos antes, em 1981.

\section{Considerações finais}

Por meio de testes econométricos de séries temporais e análises decílicas, este trabalho testou a aplicabilidade da hipótese de Kuznets - que afirma que a relação entre desigualdade na distribuição de renda e crescimento econômico segue o padrão geométrico de U-invertido, com deterioramento absoluto das rendas dos grupos menos favorecidos nos estágios iniciais do processo de decrescimento - mediante dados representativos de crescimento e desigualdade de renda para o Brasil, no período 1976-2007.

A análise dos decis, separadamente nos três subperíodos (1981/89, 1989/99 e 1999/07) corrobora os resultados das estimações realizadas nos capítulos anteriores do presente trabalho, de modo a fornecer subsídios à explicação do comportamento sistemático da relação de longo prazo entre crescimento econômico e desigualdade distributiva da renda no Brasil, sob o âmbito teórico da hipótese da Curva de Kuznets.

De modo geral, as evidências encontradas permitem afirmar que o comportamento médio da estrutura distributiva no Brasil ao longo do período 1976-2007 segue um padrão onde se verificam, nos estágios iniciais do desenvolvimento, elevações na renda da parcela mais rica da população à custa de reduções absolutas na renda dos grupos mais pobres, provocando aumentos sucessivos da desigualdade até um ponto máximo, a partir do qual 
o movimento se inverte, inicialmente por conta dos ganhos absolutos de renda dos mais pobres à custa do grupo mais rico e depois com ganhos absolutos de ambos os grupos, porém, proporcionalmente maiores para os grupos de renda mais baixa. Tais resultados estão, portanto, em perfeito acordo com a hipótese de Kuznets.

\section{Referências bibliográficas}

AHLUWALIA, M. S. (1976). "Income distribution and development; some stylized facts." American Economic Review, 66; p. 128-135.

JACINTO, P. A.; TEJADA, C. A. O. (2004). "Desigualdade de Renda e Crescimento Econômico nos Municípios da Região Nordeste do Brasil: O que os dados têm a dizer?” In: XXXII Encontro Nacional de Economia, Paraíba.

KUZNETS, S. (1992). Crescimento Econômico Moderno. Rio de Janeiro: Abril Cultural.

KUZNETS, S. (1955). "Economic Growth and Income Inequality". American Economic Review, 45; p. 1-28.

ROBINSON, S. (1976). "A note on the U hypothesis relating income inequality and economic development.” American Economic Review, 66.

SALVATO, M. A. et al. (2006). "Crescimento e Desigualdade: evidências da Curva de Kuznets para os municípios de Minas Gerais - 1991/20oo”, Ibmec/MG, (Working Paper - WP33).

ZILBERMAN, E. (2004)..Os efeitos da desigualdade no crescimento. [Monografia] Prêmio IPEA 40 anos.

Recebido em: 19 de maio de 2010

Aceito em: 17 de agosto de 2010 